\title{
TikTok: ¿una nueva herramienta educativa para combatir la COVID-19?
}

\section{TikTok: $A$ new educational tool in the fight against COVID-19?}

\section{Correspondencia Naysha Becerra Chauca nayshabecerra0401@gmail.com}

Recibido: 08/06/2020 Aprobado: 15/06/2020

Citar como: Becerra-Chauca N, Taype-Rondan A. TikTok: ¿una nueva herramienta educativa para combatir la COVID-19? Acta Med Peru. 2020;37(2):249-51. doi: https://doi.org/10.35663/ amp.2020.372.998

\section{Naysha Becerra-Chauca ${ }^{1, a}$, Alvaro Taype-Rondan ${ }^{2, b}$}

Facultad de Salud Pública y Administración, Universidad Peruana Cayetano Heredia. Lima, Perú.

Unidad de Investigación para la Generación y Síntesis de Evidencias en Salud, Universidad San Ignacio de Loyola. Lima, Perú. Licenciada en Obstetricia; ${ }^{b}$ médico cirujano, magíster en Epidemiología.

\section{Sr. Editor,}

TikTok es una red social lanzada al mercado chino en 2016 (como Douyin) y al internacional en 2017 (como TikTok). En 2018, fue la aplicación móvil más descargada en Estados Unidos, y actualmente está disponible en más de 150 países y tiene más de 800 millones de usuarios activos mensuales. El 41\% de ellos tienen entre 16 y 24 años, una población más joven de los que encontramos en otras redes sociales ${ }^{[1,2]}$.

TikTok permite crear y compartir videos cortos (15 a 60 segundos) de fácil y rápida edición con una gran variedad de efectos y sonidos incluidos en su galería (alimentada por los propios usuarios) ${ }^{[2]}$. Una de las características de esta aplicación es el uso de la tecnología Al (Artificial intelligence, por sus siglas en inglés), por el cual, el algoritmo aprende rápidamente las preferencias del individuo al capturar los "me gusta», comentarios y el tiempo que ven los videos ${ }^{[3]}$. Sin embargo, no es necesario subir videos a la aplicación ni «seguir» (solicitar información continua) a un usuario determinado para mantener una interacción activa, pues apenas se ingresa a la aplicación comienzan a mostrarse videos de diversos usuarios que puedes compartir, incluso en otras redes sociales. El contenido de los videos suele ser humorístico en su mayoría.

La pandemia por COVID-19 y la necesidad de distanciamiento físico ha mantenido a las personas en sus casas, lo que ofreció una oportunidad para explorar esta aplicación, que antes era utilizada principalmente por adolescentes. En marzo, las descargas de TikTok alcanzaron 75 millones, un 25\% más que las descargas de febrero de $2020^{[4]}$.

Asimismo, diversas personas e instituciones buscan brindar información sobre COVID-19 a través de TikTok. Esto se evidencia por un creciente número de videos sobre el tema en esta red social, algunos de los cuales incluyen retos (challenges en inglés) con hashtags cómo \#stayathomechallenge (que alcanzó 872 millones de visualizaciones) o \#safehandschallenge (36,5 millones de visualizaciones), para animar a replicar estos comportamientos. Un claro ejemplo es el \#Ghencovychallenge, o más conocido como \#washyourhandschallenge, un video que presenta a dos bailarines vietnamitas haciendo una coreografía y mostrando la correcta forma de lavado de manos ${ }^{[5]}$.

Sin embargo, algunos videos pueden contener información incorrecta, como se ha evidenciado en otras redes sociales ${ }^{[6]}$, lo que puede perjudicar la salud de los usuarios al generarles mayor ansiedad y estrés ${ }^{[7]}$. Debido a ello, TikTok actualmente presenta información confiable de instituciones oficiales como la Organización Mundial de la Salud (OMS) y agencias de las Naciones Unidas (UN) en una pestaña exclusiva para COVID-19, que está presente permanentemente en la esquina superior derecha de todos los videos; así como un enlace en la parte inferior de los videos que contiene información sobre COVID-19. Este espacio incluye videos de siete cuentas oficiales, además de enlaces para lecturas 
Tabla 1. Características de las cuentas oficiales de instituciones internacionales que aparecen bajo la pestaña de COVID-19 en TikTok.

\begin{tabular}{|c|c|c|c|c|c|}
\hline \multirow{2}{*}{$\begin{array}{l}\text { Institución que } \\
\text { representa } \\
\text { (Nombre de cuenta) }\end{array}$} & \multirow{2}{*}{$\begin{array}{l}\text { Fecha de primer } \\
\text { video publicado }\end{array}$} & \multirow{2}{*}{$\begin{array}{l}\text { Fecha del } \\
\text { primer video } \\
\text { sobre COVID-19 }\end{array}$} & \multicolumn{3}{|c|}{ Video más visto sobre COVID-19 } \\
\hline & & & Fecha & $\begin{array}{c}\mathrm{N} \text { de vistas } \\
\text { (N de me gusta)* }\end{array}$ & Tema \\
\hline $\begin{array}{l}\text { World Health } \\
\text { Organization } \\
\text { (who) }\end{array}$ & $28 / 02 / 2020$ & $28 / 02 / 2020$ & $29 / 05 / 2020$ & $\begin{array}{c}54,5 \mathrm{M} \\
(521,7 \mathrm{~K})\end{array}$ & $\begin{array}{c}\text { El consumo de tabaco } \\
\text { como factor de riesgo para } \\
\text { COVID-19 }\end{array}$ \\
\hline $\begin{array}{l}\text { The UN Migration Agency } \\
\text { IOM } \\
\text { (unmigration) }\end{array}$ & $28 / 01 / 2020$ & 02/03/2020 & $02 / 03 / 2020$ & $\begin{array}{c}20,4 \mathrm{M} \\
(296,0 \mathrm{~K})\end{array}$ & $\begin{array}{l}\text { No discriminación contra } \\
\text { los migrantes durante la } \\
\text { pandemia de COVID-19 }\end{array}$ \\
\hline $\begin{array}{l}\text { International Federation } \\
\text { of Red Cross } \\
\text { (Ifrc) }\end{array}$ & 29/05/2019 & $22 / 01 / 2020$ & $02 / 03 / 2020$ & $\begin{array}{c}32,1 \mathrm{M} \\
(992,3 \mathrm{~K})\end{array}$ & $\begin{array}{l}\text { Desmentir rumor sobre el } \\
\text { potencial de las secadoras } \\
\text { de mano para eliminar } \\
\text { coronavirus }\end{array}$ \\
\hline $\begin{array}{l}\text { The World Economic } \\
\text { Forum } \\
\text { (worldeconomicforum) }\end{array}$ & 03/09/2019 & $10 / 02 / 2020$ & $28 / 02 / 2020$ & $\begin{array}{l}43,3 \mathrm{M} \\
(2,0 \mathrm{M})\end{array}$ & Formas de contagio \\
\hline $\begin{array}{l}\text { The UN Refugee Agency } \\
\text { (refugees) }\end{array}$ & $14 / 11 / 2019$ & $10 / 03 / 2020$ & $24 / 03 / 2020$ & $\begin{array}{c}14,0 \mathrm{M} \\
(483,1 \mathrm{~K})\end{array}$ & $\begin{array}{l}\text { Los refugiados y su } \\
\text { importante aporte en la lucha } \\
\text { contra COVID-19 }\end{array}$ \\
\hline $\begin{array}{l}\text { UN Food Agency } \\
\text { (FAO) }\end{array}$ & 29/11/2019 & 05/03/2020 & $05 / 03 / 2020$ & $\begin{array}{l}34,4 \mathrm{M} \\
(1,3 \mathrm{M})\end{array}$ & $\begin{array}{l}\text { Formas de saludo sin tocarse } \\
\text { las manos }\end{array}$ \\
\hline $\begin{array}{l}\text { UN Women } \\
\text { (unwomen) }\end{array}$ & $4 / 03 / 2020$ & 07/04/2020 & $07 / 04 / 2020$ & $\begin{array}{l}279 \mathrm{~K} \\
(15 \mathrm{~K})\end{array}$ & $\begin{array}{l}\text { Visibilidad de mujeres en } \\
\text { primera línea contra el } \\
\text { COVID-19 }\end{array}$ \\
\hline
\end{tabular}

* Subidos hasta el 22 de junio de 2020

M: millones; K: miles; UN: United Nations; IOM: International Organization for Migration

sobre medidas de protección, mitos, y una sección de preguntas/ respuestas. Las cuentas oficiales comenzaron a publicar videos entre el 29 de mayo de 2019 y el 28 de febrero de 2020, lo cual nos indica su reciente incursión en TikTok, incluso una de ellas inició su actividad con la pandemia ${ }^{[8,9]}$ (Tabla 1).

El video más visto de estas cuentas fue subido el 29 de febrero y tuvo 54,5 millones de visualizaciones, 521,7 miles de «me gusta» y fue compartido más de 12 mil veces. Estas cifras, nos revelan un gran alcance del contenido de estas cuentas oficiales. Sin embargo, aún están lejos de los videos más populares de TikTok, que han logrado 219 millones de visualizaciones y 24 millones de «me gusta» ${ }^{[1]}$. Esto evidencia el reto de combinar lo que los usuarios esperan en TikTok (posiblemente videos cortos, humorísticos y replicables) con contenido educativo, para un mayor alcance.

El crecimiento del número de descargas con un grupo etario variado, el compromiso de TikTok en la lucha contra la desinformación, y el potencial de alcance evidenciado en las cuentas activas con información oficial, convierten a esta aplicación en una ventana de oportunidad para educar en salud, especialmente en tiempo de pandemia y confinamiento. Sin embargo, no hemos hallado estudios que evalúen su real efecto. Por ende, resulta importante realizar estudios para responder preguntas que nos permitan optimizar su impacto, como: ¿Qué videos van a tener mayor número de visualizaciones, «me gusta» y compartidas?, ¿'Los challenges promueven la replicación del comportamiento?, ¿La información brindada en estos videos tendrá impacto en el comportamiento humano en salud, y de qué magnitud? y ¿Qué otras alternativas existen para reducir la desinformación y su impacto?

Contribuciones: ambos autores se encargaron de la concepción de la carta, análisis e interpretación de los datos, de la revisión crítica y aprobación de la versión final. NBC se encargó de adquisición de los datos y la redacción de la primera versión de la carta.

Potenciales conflictos de interés: los autores declaran no tener conflictos de interés.

Fuente de financiamiento: autofinanciado.

\section{ORCID:}

Naysha Becerra-Chauca: https://orcid.org/0000-0001-5706-7351 Alvaro Taype-Rondan: https://orcid.org/0000-0001-8758-0463 


\section{REFERENCIAS BIBLIOGRÁFICAS}

1. Omnicore Group. TikTok by the Numbers: Stats, Demographics \& Fun Facts [Internet]. OMNICORE; 2020 [citado el 28 de abril de 2020]. Disponible en: https://www.omnicoreagency.com/tiktokstatistics/.

2. Zhu C, Xu X, Zhang W, Chen J, Evans R. How health communication via Tik Tok makes a difference: a content analysis of Tik Tok accounts run by Chinese Provincial Health Committees. International journal of environmental research and public health. 2020;17(1):192. doi: 10.3390/ijerph17010192.

3. David J. The TikTok Strategy: Using AI Platforms to Take Over the World Pensilvania [Internet]. INSEAD the bussiness school for the world; 2019 [citado el 28 de abril de 2020]. Disponible en: https:// knowledge.insead.edu/entrepreneurship/the-tiktok-strategyusing-ai-platforms-to-take-over-the-world-11776.

4. Amlôt M. Coronavirus: TikTok reports record growth as mobile use soars under lockdown [Internet]. Al Arabiya; 2020 [citado el 28 de abril de 2020]. Disponible en: https://english.alarabiya.net/ en/coronavirus/2020/05/07/Coronavirus-TikTok-records-recordgrowth-as-mobile-use-soars-under-lockdown.
5. Burke C. How To Do TikTok's Coronavirus Hand Washing Challenge [Internet]. BUSTLE; 2020 [citado el 28 de abril de 2020]. Disponible en: https://www.bustle.com/p/how-to-do-tiktoks-coronavirushand-washing-challenge-22606497.

6. Li HO-Y, Bailey A, Huynh D, Chan J. YouTube as a source of information on COVID-19: a pandemic of misinformation?. BMJ Global Health. 2020;5(5):e002604. doi: 10.1136/bmjgh-2020-002604

7. Sahoo SS, Sahu DP, Kankaria A. Mis-infodemic: The Achilles' heel in combating the COVID-19 pandemic in an Indian perspective. Monaldi archives for chest disease. 2020;90(2). doi: 10.4081/ monaldi.2020.1405.

8. Harrison A. The WHO Associates With TikTok to Restrain the COVID-19 Misinformation Transmission 2020 [Internet]. Digital Marketing Community; 2020 [citado el 28 de abril de 2020]. Disponible en: https://www.digitalmarketingcommunity.com/ news/tiktok-covid-19/.

9. TikTok. Supporting Our Community Through COVID-19 [Internet]. TikTok; 2020 [citado el 28 de abril de 2020]. Disponible en: https:// www.tiktok.com/safety/resources/covid-19. 